\title{
Music That Changed My Life. POP-MUSIK UND SELBSTVERSTÄNDNIS
}

\author{
Matthias Vogel
}

»I think it was a day or two after that that his plane went down. And somebody - somebody I'd never seen before handed me a Leadbelly record with the song >Cottonfields $<$ on it. And that record changed my life right then and there. Transported me into a world I'd never known. [...] I must have played that record a hundred times « (Bob Dylan 2017). ${ }^{1}$

Glaubt man empirischen Untersuchungen, so wird heute mit Abstand die meiste Musik nicht im Konzertsaal oder gar anlässlich von Hausmusikabenden gehört, sondern im Auto, in der Bahn, im Flugzeug, beim Einkaufen oder bei der Arbeit (Huron 2001: 51). Und es ist nicht rituelle oder Kunstmusik, die in diesen Kontexten ganz überwiegend gehört wird, sondern Pop-Musik - sei es, um Langeweile oder das Gefühl der Einsamkeit zu vertreiben, sich vor einem Wettkampf zu pushen oder besser auf die Arbeit konzentrieren zu können. So gesehen wäre Pop-Musik ein ziemlich nebenwirkungsarmes Mittel der Selbstregulation. Das ist richtig, greift meines Erachtens aber zu kurz. Denn darüber hinaus spielt Pop-Musik im Leben vieler Menschen offenbar eine Rolle, die eng mit der Entwicklung ihres Selbstverständnisses, damit, welche Person sie sein oder werden wollen, verknüpft ist.

Auf den ersten Blick harmoniert diese Ansicht gut mit der Perspektive, die Tia DeNora in ihren einflussreichen Arbeiten auf die Musik eingenommen hat. Doch während DeNora beeindruckende Evidenz dafür liefert, dass Musik eine instrumentelle Rolle in Prozessen der Selbstregulation und -situierung spielt, bleibt sie eine Erklärung dessen, was ausgerechnet Musik und im Besonderen Pop-Musik für die »Konstitution von Identität « oder - wie ich lieber sagen würde - die Entwicklung und Transformation der personalen Identität geeignet macht, letzten Endes schuldig. Dafür scheint mir nicht zuletzt DeNoras Verschiebung des theoretischen Fokus von der Frage, „what music

1 Dylan bezieht sich auf den Flugzeugabsturz, bei dem u.a. Buddy Holly starb. Für den Hinweis auf die Rede danke ich Ralf von Appen. 
>means«« zur Frage, »what it >does« (DeNora 1999: 34) verantwortlich zu sein.

Ich möchte demgegenüber versuchen, eine solche Erklärung zu geben. Dazu ist es nötig, den Fokus neu einzustellen und etwas auszuholen. Zunächst, im ersten Abschnitt, votiere ich dafür, dass die Frage, warum wir Musik hören, nicht nur legitim ist, sondern einer Antwort bedarf, die sowohl über die meines Erachtens unverständliche Antwort »um ihrer selbst willen« als auch über die zu kurz greifende Antwort »um ihrer Effekte willen« hinausgeht. Im zweiten Abschnitt präsentiere ich eine Reihe von Äußerungen, in denen Hörer behaupten, dass ein bestimmtes Musikstück, die Musik einer Band oder einer bestimmten Stilrichtung ihr Leben verändert hat. Da die materiellen Lebensbedingungen von diesen Veränderungen unberührt geblieben sein dürften, können wir vermuten, dass sie sich in Dimensionen vollzogen haben, die für ihr Selbstverständnis eine gewisse Rolle spielen. Auch wenn Hörer in aller Regel keine Erklärungen für derartige Prozesse geben und sich mit Blick darauf sogar ratlos zeigen, finden sich in ihren Selbstauskünften doch Andeutungen dessen, was hier zum Tragen kommen könnte. Der dritte Abschnitt skizziert dann die Grundlinien einer Theorie musikalischen Verstehens in Begriffen einer Theorie des Nachvollzugs. Im vierten Teil versuche ich, die vorangegangenen Überlegungen zu einer Antwort auf die Frage zusammenzuführen, warum insbesondere Pop-Musik ein leistungsfähiges Medium der Entwicklung und Transformation von Selbstbildern ist. ${ }^{2}$ Das - so meine These - lässt sich verständlich machen, wenn die Relationen, die Subjekte zu sich und zur Musik einnehmen, als Relationen des Verstehens aufgefasst werden.

2 In meinem Leben hat es nicht wenige solcher musikimprägnierten Veränderungsprozesse gegeben. So haben die Alben von Joy Division meine Perspektive auf mich und die Welt über viele Monate bestimmt, es mir erlaubt, eine Haltung zu den sich verdüsternden Verhältnissen der frühen 1980er einzunehmen, die gegen jede weitere Enttäuschung gefeit zu sein schien, weil Depression nichts mehr war, das man noch auf Distanz hätte halten müssen, sondern etwas, das geeignet war, einen Unterschied zu denen zu machen, die das Debakel nicht verstanden oder BWL studierten, etwas, zu dem man Tanzen konnte (»Isolation«) oder sich mit dem schweren Glanz einer verdunkelten Sonne verbinden konnte, die am Ende von »Day Of The Lords « über der ganzen Szenerie aufsteigt. Techno und House stand ich viele Jahre später zunächst als Musik gegenüber, die mir dumm erschien und nichts sagte, bis zu einem Abend in einem Hamburger Club, der einem Saulus/Paulus-Erlebnis gleichkam, das mir den Zugang zu der wortlosen Feier gewährte, die seit nunmehr 25 Jahren anlässlich der Hochzeit von Tanz und (universeller) Maschine gefeiert wird und meinen Blick auf Urbanität und Zivilisation neu grundiert hat. 


\section{Nicht um ihrer selbst willen}

Ich habe Zweifel an der Auffassung, dass wir Musik (wie die Kunst insgesamt) um ihrer selbst willen schätzen (vgl. dazu Vogel 2013b), und diese Zweifel hat DeNora in ihrem vielbeachteten Aufsatz »Music as a Technology of the Self« (1999) noch weiter genährt. Ihre soziologischen Untersuchungen erweisen nämlich Musik als ein inmitten unserer alltäglichen praktischen Lebensvollzüge situiertes Phänomen, das wir nicht nur für Prozesse der emotionalen Selbstregulation und für soziale Rituale nutzen, sondern auch als eine kulturelle Ressource für Prozesse der Selbstkonstruktion. Das läuft auf die These hinaus, dass Musik eine wichtige Rolle in Prozessen spielt, in denen wir Antworten auf die Frage entwickeln, wie die Person beschaffen ist, die wir sind oder sein wollen: als Mittel der Selbstinterpretation, der Artikulation eines Selbstbilds und der Selbstsituierung. ${ }^{3}$

DeNora kann mittels ihrer Interviews zweifelsfrei belegen, dass Menschen Musik in alltäglichen Kontexten zu Zwecken der emotionalen Selbstregulation nutzen. Dass Musik für solche Zwecke geeignet ist, lässt sich im Rekurs auf die kausalen Effekte der Musik (»what it does «) gut erklären. ${ }^{4}$ Musik kann sich als etwas herausstellen, das energetisierende, beruhigende, fokussierende oder euphorisierende Effekte zeitigt; und wir Rezipienten, die wir ein Wissen über ebensolche Effekte auf uns selbst erworben haben, können Musik instrumentell zur erneuten Herstellung dieser Effekte nutzen. Daraus folgt, dass wir in der Perspektive unseres musikalischen Appetits antizipieren können, nach welcher Musik uns der Sinn steht, welche Musik uns jetzt zum Beispiel guttäte.

3 Ich vermeide im Folgenden Ausdrücke wie »Selbstkonstitution «, »Selbstkonstruktion « oder »Konstitution von Identität « (derer sich DeNora häufig bedient), weil sie systematisch missverständlich sind: Wenn das "Selbst « (?) als das Subjekt der Konstitution verstanden wird, dann gibt es wohl keinen Prozess, der diese Bezeichnung zu Recht trägt, denn alle Konstitutionsprozesse haben Bedingungen, die nicht zum »Selbst « gehören und nicht in der Verfügungsgewalt der Person stehen. Und was soll das sein, ein »Selbst«? »Konstruktion« hat m.E. deutlich zu voluntaristische Konnotationen; und »Identität« muss nicht konstituiert werden, weil jedes Ding trivialerweise mit sich selbst identisch ist. Ganz ohne diese Ausdrücke, die geeignet sind, unseren Verstand zu verhexen, komme ich aber auch nicht aus: Mit dem Selbstverständnis einer Person meine ich die Antwort, die sich eine Person auf die Frage gibt, was für sie selbst spezifisch ist oder spezifisch sein soll.

4 Auch wenn die Frage interessant ist, warum Musik solche Effekte herbeizuführen vermag, hängt die Plausibilität der Beobachtungen DeNoras natürlich nicht davon $a b$, dass wir eine Antwort auf diese Frage haben. Ergiebige Überlegungen zu einer derartigen Theorie finden sich m.E. in Trevarthen (2000). 
Auch wenn in derartige Techniken der Selbstregulation ein Wissen über uns eingeht, scheint mir dieses Wissen nicht über ein empirisches Wissen über unsere psychischen Dispositionen hinauszugehen, und nur in diesem trivialen Sinn von uns als Personen zu handeln. Ebenso wie ich wissen kann, dass mir nachmittags grüner Tee meist besser tut als schwarzer Kaffee, kann ich wissen, dass mir Substrata von Biosphere bei der Schreibtischarbeit besser tut als Stadt der Angst von Turbostaat. Aber solches Wissen macht nicht verständlich, warum Musik in einem nichttrivialen Sinn für Prozesse der Bestimmung der eigenen Person geeignet ist. Ähnlich verhält es sich, wenn wir uns von den blank kausalen Effekten lösen und zum Beispiel Relationen des Evaluierens von Musik betrachten. Natürlich können wir Musik dazu nutzen, uns als Personen zu beschreiben, die dieses Stück, diese Band, diesen Stil mögen, ablehnen oder gar abscheulich finden. Aber dazu lassen sich natürlich auch Automarken, Mode, Designstile oder Landschaften nutzen. Interessant ist jedoch nicht die Tatsache, dass jemand Siouxsie and the Banshees, U2 oder Moby schätzt, sondern die Tatsache, dass dieses Wertschätzen Signifikanz für die jeweiligen Personen hat oder gewinnen kann. Den Unterschied, um den es hier geht, kann man sich veranschaulichen, wenn man folgende Äußerungen vergleicht:

(a) Ich bin eine Person, die Muskat mag.

(b) Ich bin eine Person, die Run the Jewels mag.

Während in (a) ein Faktum artikuliert wird, dessen Erwähnung eine Liste kontingenter Präferenzen verlängern könnte, die wir achselzuckend zur Kenntnis nehmen, ist die Wertschätzung, die in (b) ausgedrückt wird, dazu geeignet, etwas Interessantes über eine Person zu offenbaren, und zwar auch für die Person selbst. Was wir angesichts dieses Kontrasts verstehen müssen, ist die Quelle der Signifikanz von (b). Diesbezüglich besteht ein erster Schritt darin, sich zu vergegenwärtigen, dass aus (a) nichts weiter folgt: Die Tatsache, dass jemand Muskat mag, ist kein guter Prädiktor dafür, dass er Schokolade mag, Audi fährt oder Vegetarier ist. Die Muskat-Präferenz scheint mit unendlich vielen anderen vereinbar zu sein, sodass es weder überraschend wäre zu erfahren, dass jemand, der Muskat mag, auch Vanille schätzt, noch dass er außerdem eine Vorliebe für englische Parks hat.

Mit Blick auf die gesuchte Quelle der Signifikanz von (b) können wir uns auf die Tatsache stützen, dass das Wertschätzen eines Musikstücks oder der Musik einer Band oder eines Stils eine Aktivität der wertschätzenden Person voraussetzt, die tatsächlich etwas über die Person sagt. Sie ist eine Person, der die Musik etwas sagt, eine Person, der es gelingt, die Musik zu verstehen, und das heißt: auf eine Weise zu hören, die ihren Sinn erfasst. Hier verhält 
es sich anders als im Falle des Muskat-Liebhabers: Es würde uns doch sehr überraschen, wenn sich in der Sammlung unseres Run the Jewels-Fans auch alle Alben von Mr. President oder Sarah Connor befänden. Nicht dass es nicht möglich wäre, alle drei zu schätzen, aber die Tatsache, dass jemand das tut, macht zumindest neugierig darauf, was das wohl für eine Person sein mag.

Das bringt mich zu meinem eigentlichen Thema und meiner zentralen Idee, dass nämlich der Zusammenhang zwischen Pop-Musik und personaler Identität genauer erläutert werden kann, wenn die Relationen, die ein Subjekt zu sich selbst einnimmt, und die Relation, die es zur Musik einnimmt, als Relationen des Verstehens rekonstruiert werden.

Mit Blick auf einen recht unspezifischen Aspekt lässt sich diese Idee vielleicht am leichtesten plausibilisieren. Wahrscheinlich ist die Zahl derer, die im Erwachsenenalter gern Kinderlieder hören, recht gering. Das dürfte auch damit zusammenhängen, dass viele Kinderlieder sehr einfach strukturiert sind. Analoges gilt sicher auch für viele sogenannte Schlager. Wenn ich Schlager mag, kann das also als ein Indiz dafür genommen werden, dass ich beim Musikhören mit minimalen Herausforderungen zufrieden bin. Wer hingegen schnell am Ohrwurmcharakter eines Stücks leidet, mag simple Musik als Ursprung solchen (ästhetischen) Leidens verachten, und Personen, die solche Musik schätzen, können einem fremd werden. In dieser Hinsicht können Unterschiede in der Wertschätzung auch zu sozialen Distinktionsmerkmalen werden. Wichtig ist: Diese Unterschiede bestehen nicht nur mit Blick auf die Komplexität von Musik, sondern auch mit Blick auf die Gesten, die diese den Hörern (auch angeleitet durch die prototypische Aufführung der Gesten durch die Musiker) nahelegt. Während dem einen der Gitarrenmachismo peinlich oder zuwider ist, schätzt eine andere gerade die implizite Inszenierung von Stärke oder Macht. Interessant werden diese Differenzen, wenn sie im Lebensverlauf ein und derselben Person auftreten, so dass sie über Musik, die sie früher sozusagen geatmet hat, heute nur noch den Kopf schütteln kann. So mag die einst als spannend oder gar als Offenbarung empfundene polymorph-perverse Inszenierung von sexuellem Begehren in Madonnas »Justify My Love« heute nur noch albern, schematisch oder kühl kalkuliert wirken. Und noch interessanter wird es, wenn sich diese individualbiographischen Differenzen und Distanzierungen allein aufgrund musikalischer Merkmale auftun. Dem werde ich nun im nächsten Abschnitt genauer nachgehen. 


\section{Selbstauskünfte}

Wenn Musik als Musik, also qua ihrer musikalischen Eigenschaften, einen wichtigen Einfluss auf das Leben ihrer Hörerinnen nehmen kann und in irgendeiner Weise zu Prozessen des Selbstverstehens beizutragen vermag, dann sollte es Berichte oder Erzählungen geben, in denen Hörerinnen auf derartige Prozesse Bezug nehmen. Für unseren Zusammenhang sind dabei insbesondere solche Berichte von Interesse, in denen es zum einen speziell um Pop-Musik geht, die zum anderen (weitgehend) ohne Texte auskommt. ${ }^{5}$ Natürlich müssen sich lebensverändernde Effekte auch im Falle von Musik mit Texten nicht allein sprachlicher Botschaften verdanken; um die Rolle der Musik für solche Transformationsprozesse jedoch unabhängig vom Einfluss anderer Faktoren studieren zu können, lohnt es sich, die Untersuchung auf Instrumentalmusik zu beschränken, sodass wir ihren Einfluss wie in einer Laborsituation isolieren können. Wie ein Blick auf Kommentarplattformen zeigt, gibt es erstaunlicherweise keinen Mangel an Berichten über die lebensverändernde Kraft instrumentaler Pop-Musik.

Beginnen wir mit dem Nutzer Peter Grimmer, der über das 1994 erschienene Album Global Chillage von The Irresistible Force Folgendes schreibt: »| bought this on Cassette at gramaphone in chicago when $i$ was in high school. changed my life. ${ }^{6}{ }^{\circ} \gg$ Changed my life - wie kann das sein? Wie kann das Hören eines Ambient-Albums das Leben eines seiner Hörer verändern? Zumal die Stücke auf dem Album (abgesehen von ein paar Loops) keinen Text haben, der irgendeine lebensverändernde Botschaft transportieren könnte (»Macht kaputt, was euch kaputt macht!«), und Morris Gould (aka Mixmaster Morris), der sich hinter dem Pseudonym The Irresistible Force verbirgt, gewiss kein Star ist, dessen öffentliche Inszenierungen zur Identifikation einladen. Auch

5 Diedrich Diederichsen betrachtet Pop-Musik mit guten Gründen als einen Komplex aus »Bildern, Performances (meist populärer) Musik, Texten und an reale Personen geknüpften Erzählungen« (Diederichsen 2014: XI). Ich verwende hier den Ausdruck »Pop-Musik«, um den musikalischen Bestandteil dieses Komplexes herauszugreifen, und spreche dann, wenn es mir um den ganzen Zusammenhang geht, vom Pop-Musik-Komplex. Um fokussiert die Rolle der Musik zu untersuchen, ziehe ich im Folgenden darüber hinaus nur Pop-Musik heran, die weitgehend ohne Text auskommt und bei der die anderen Komponenten des Komplexes von vergleichsweise geringer Bedeutung sind.

6 Siehe den Kommentar zu »The Irresistible Force, Global Chillage [Full Album] «, www.youtube.com/watch?v=sAw_yllpSYQ, Zugriff: 10.4.2017. Mir ist bewusst, dass die Wendung »changed my life « hin und wieder auch als leicht übertriebenes Lob verwendet wird. In den von mir herangezogenen Zitaten scheint mir das jedoch nicht der Fall zu sein. Ich nehme sie daher für bare Münze bzw. als wörtlich wahre Selbstauskünfte. 
als politisches Manifest erscheint mir die Platte eher ungeeignet, selbst wenn hbmf auf discogs.com schreibt, sie sei »subversively political «. ${ }^{7}$

Eine Weise, Peters Kommentar zu deuten, besteht darin, ihn als Erwähnung einer singulären, das heißt strukturell unwiederholbaren Erfahrung zu verstehen, die beispielsweise durch eine gesteigerte meditative Aufmerksamkeit gekennzeichnet war. Gegen diese Deutung spricht jedoch die Wahl des Veröffentlichungsortes; Peter hat die Beschreibung seiner Erfahrung nicht seinem Tagebuch anvertraut, ${ }^{8}$ er hat sich vielmehr für ein öffentliches Forum entschieden, was darauf hindeutet, dass er andere Hörer auf dieses Album aufmerksam machen und dabei auf etwas hinweisen möchte, das auch für andere von Interesse sein könnte: die Tatsache, dass ihm die Musik - also etwas intersubjektiv Zugängliches - eine Art von Erfahrung ermöglicht hat, die sein Leben verändert hat. Da Global Chillage weder Peters Kontostand verändert hat noch via Text seine Einstellung zur Welt, stehen uns als einzige Ressource für die Veränderung in Peters Leben diejenigen ästhetischen Erfahrungen zur Verfügung, die er anlässlich oder mit der Musik gemacht hat. Und da ästhetisches Erfahren ein Prozess ist, an dem Rezipienten selbst einen kreativen Anteil haben, können wir vermuten, dass es Peter vielleicht gelungen ist, beim Hören von Global Chillage eine der Musik angemessene Form der Aufmerksamkeit zu entwickeln, die ihn in die Lage versetzte, eine Musik, die in den Ohren vieler wie formloses echogetränktes repetitives Geklimper klingt, als eine (musikalische) Einheit zu hören, und ihm fortan auch in anderen Kontexten zur Verfügung stand. Vielleicht evoziert die Musik bei ihm die Vorstellung einer zyklischen Geschäftigkeit, die den Kontext all unserer Lebensvollzüge bildet, vielleicht hat ihn aber auch die subkutane Musikalisierung des geräuschhaften Hintergrunds in den Bann geschlagen. Entscheidend ist jedenfalls, dass die Weise, in der die Musik zu einer Veränderung von Peters Leben beigetragen hat, mutmaßlich die Form einer Veränderung seiner Erfahrungsfähigkeit hat - eine Art Horizonterweiterung, die sich darin zeigt, dass sich ihm eine neue Art von Musik erschlossen hat. Wenn das der Fall ist,

7 www.discogs.com/de/Irresistible-Force-Global-Chillage/master/30708, Zugriff: 10.4.2017.

8 Es gibt zahllose derartige Kommentare, nicht wenige nach dem Muster »i listened to this on so much acid. i felt like the hubble telescope. such wonders ..." (vgl. Ken, ebd.). Obwohl es interessant ist, dass Musikstücke es so effektiv vermögen, Erinnerungen zu aktivieren - der Chartshow-Industrie ist ihr Publikum sicher! -, helfen uns Memes wie »Gets me back to those days«, »amazing blast from the past«, »memories! « nicht wirklich weiter. Denn einerseits - man setze sich in einen VW-Käfer oder Trabant - können das auch alte Autos und natürlich Fotos, und andererseits ist die Tatsache, dass Musik uns so effektiv in die Vergangenheit versetzen kann, gerade selbst erklärungsbedürftig. 
dann hätte er die Erfahrung, die zu dieser Veränderung geführt hat, womöglich auch mit Musiken ähnlichen Typs, zum Beispiel Brian Enos Music for Airports oder Chill Out von The KLF, machen können.

Es ist interessant, dass die Gründe und Hinsichten, in denen Musik zu einer Veränderung des Lebens beigetragen hat, selten artikuliert werden. So kann sich Genki78 zwar noch ganz genau an die Umstände des ersten Kontakts mit »Melt« von Leftfields Album Leftism erinnern, aber warum genau das Stück eine so starke Faszination auf ihn/sie ausübte, schreibt er/sie nicht:

">Melt< (track 3) was a life changing experience! Never heard such a fascinating ambient tune before that. I heard it for the first time during vacation in Belgium in the mid-90s, when going into a clothing shop near the beach, and they played »Melt « [...]. Then we asked the sales girl which song they are currently playing, because it sounds awesome! And they showed us the Leftism CD. We never heard about Leftfield before. That was such a great thing! «9

Wie schwierig es ist, die lebensverändernde Kraft musikalischer Erfahrungen zu artikulieren, macht - vorangegangene Statements aufnehmend - XXNovaXxs Kommentar zu Goldies »Timeless« explizit:

»Groundbreaking, Utter brilliance... Okay okay.. This album is Fucking insanely brilliant.. In my opinion the stand out here is »Timeless «. I get a mysterious and unusual feel of nostalgia every time i hear those lush pads and Diane's soothing voice. Timeless got me into DNB [Drum and Bass], and it is and probably will remain my favourite track of all time, among a couple of others. [...]. Thank you Goldie for changing my life, it's a hard thing to put into words of what this album has done to me, but I m sure you can figure it out. Absolutely jaw dropping. «10

»It's a hard thing to put into words « - die Äußerung, dass es nicht leicht ist zu sagen, worin die transformierende Kraft besteht, ist möglicherweise ein Indiz dafür, dass mit der Rede von der Lebensveränderung mehr zum Ausdruck gebracht wird als höchste Wertschätzung. Unter dem Titel »23 Records that made me who I am« schreibt co_existence:

"Everyone has a history of records that they have been exposed to, beyond their control. I think those records shape our personality to some extent whether we like it or not. Here are 23 key records of my life.«11

9 www.discogs.com/de/Leftfield-Leftism/release/3301\#comment641903, Zugriff: 10.4.2017, meine Hervorhebung.

10 www.discogs.com/Goldie-Timeless/release/2399, Zugriff: 10.4.2017, meine Hervorhebung.

11 https://rateyourmusic.com/list/co_existence/23-records-that-made-me-who-iam, Zugriff: 10.4.2017, meine Hervorhebung. 
Hier wird - kontra »Technologien des Selbst « - die These vertreten, dass wir Musik nicht nur aktiv als Mittel der Persönlichkeitsentwicklung nutzen, sondern dass Pop-Musik auch ohne unser Zutun auf die Prozesse der Persönlichkeitsentwicklung einwirkt, also faktisch Teil jenes auditorischen Biotops sind, in dem wir geworden sind, was wir sind. Das leuchtet ein, aber unklar ist immer noch, wie Musik genau dies tut bzw. warum sie es tun kann. 5thEye deutet in seinem/ihrem Kommentar zum Ambient-Album Substrata von Biosphere eine interessante, wenngleich noch immer sehr allgemeine Hinsicht an, die in einer gewissen Spannung zur Auffassung von co_existence steht:

»The best ambient album I have ever listened to in my life. Every single track is just perfect. "Kobresia « makes me want to cry whenever I listen to it. This album has changed my outlook on life, it is just so damned beautiful. Listening to this, I feel like I am in the arctic - I know that sounds weird but it's true! Such an amazing album. $\ll 12$

Für 5thEye besteht die lebensverändernde Erfahrung darin, dass sich die Perspektive auf ihr/sein Leben verändert hat. Die musikalische Erfahrung ist nicht bloß ein kausaler Faktor, der Einfluss auf den gegenwärtigen psychischen Zustand einer Person nehmen kann, sondern eine Kraft, die zu einer Veränderung von Sichtweisen beizutragen vermag. Wie entfaltet sich diese Kraft? Unter dem Titel »Ultimate... 10 bands that make me who I am « ${ }^{13}$ veröffentlicht redhalo eine Liste von zehn Bands und stellt dem letzten Eintrag folgenden Satz voran: „I was 29 when I had my last revolution in how I listen and understand music. «14 Dieser Spur möchte ich im nächsten Abschnitt nachgehen.

12 https://rateyourmusic.com/list/5thEye/1001-albums-you-must-hear-beforeyou-die-rym-edition/3, Zugriff: 10.4.2017, Anführungszeichen und Hervorhebung von mir.

13 https://rateyourmusic.com/list/redhalo/ultimate 10_bands_that_make_me _who_i_am_, Zugriff: 10.4.2017.

14 Ebd., meine Hervorhebung. 


\section{Musik verstehen: Eine ganz kurze Einführung in die Theorie des Nachvollzugs}

Musikhören steht wie das Hören sprachlicher Äußerungen vor der Alternative, das Gehörte zu verstehen oder nicht zu verstehen. ${ }^{15}$ Im Unterschied zu dem, was wir verstehen, wenn wir sprachliche Äußerungen verstehen, sollten wir das, was wir im Falle der Musik verstehen, aber nicht als die Bedeutung eines Musikstücks betrachten. Die Bedeutung einer sprachlichen Äußerung zu verstehen, setzt nach einer weithin geteilten Auffassung voraus, dass man informativ artikulieren kann, was man verstanden hat.

Das aber ist nur möglich, wenn verschiedene Weisen zur Verfügung stehen, in denen ein und dieselbe Bedeutung ausgedrückt werden kann, denn es ergibt schlicht keinen Sinn, zu behaupten: "Ich weiß, was $x$ bedeutet, ich kann nur nicht sagen, was. «Im Fall des Musikverstehens sind diese Bedingungen jedoch nicht zu erfüllen. Denn aufgrund der unersetzbaren Rolle der sinnlichen Beschaffenheit eines erklingenden Musikstücks kann es nicht durch etwas vertreten werden, das andere sinnliche Eigenschaften, gleichwohl aber dieselbe Bedeutung hat. Klingt die vermeintliche Bedeutungsangabe anders als das Stück, kann sie das Stück nicht vertreten. Das ist bei sprachlichen Äußerungen anders. Adorno (1978: 251) hat diesen Zusammenhang so ausgedrückt, dass das mit Musik Artikulierte »sich nicht von der Musik ablösen« lässt.

Während man also die Bedeutung von »es regnet « vermittels von »it is raining « ausdrücken kann, wobei die zweite Äußerung eine Form ist, die Bedeutung der ersten anzugeben, gibt es keine derartigen informativen Übersetzungen von musikalischen Äußerungen. Musikalische Aufführungen oder musikalische Äußerungen können bestenfalls durch ihre Wiederholung vertreten werden; Wiederholungen sind jedoch keine informativen Bedeutungsangaben. Abgesehen beispielsweise von ihrem Gebrauch als Klingelton oder Sound-Logos haben Musikstücke darüber hinaus typischerweise keine repräsentationale Funktion. Wenn wir Musik als Musik und nicht bloß als Signal oder als Indikator für die Qualität eines Lautsprechers hören, dann entschlüsseln wir sie nicht, indem wir Sachverhalte identifizieren, die mit ihrer Hilfe ausgedrückt oder repräsentiert werden. Daher fehlt Musik etwas (wie beispielsweise Wahrheits- oder Erfüllungsbedingungen), woran wir den Erfolg einer stellvertretenden Übersetzung überhaupt messen könnten. Kurzum: Musik zu

15 Die nachfolgenden Gedanken zum Musikverstehen habe ich anderenorts genauer entwickelt, insbesondere in Vogel (2007) und zuletzt auf ganz ähnliche Weise wie in diesem Abschnitt in Vogel (2018). 
verstehen, heißt in aller Regel nicht, einen artikulierbaren repräsentationalen Gehalt zu erfassen. ${ }^{16}$

Will man im Lichte dieser Schwierigkeiten an der Intuition festhalten, dass Musik mehr ist als »bloßes Spielwerk der Sinne« (Hanslick 1989: 95) oder als etwas, das wir aufgrund seiner Wirkungen schätzen, nämlich etwas, das wir verstehen können, muss das, was wir beim Musikverstehen erfassen, etwas anderes als Bedeutung sein. Die Idee besteht nun darin zu sagen, dass wir nicht ihre Bedeutung, sondern ihren Sinn erfassen - indem wir ihre sinnlich erfahrbare Beschaffenheit in Akten des Nachvollzugs strukturieren. ${ }^{17}$ Ganz grob lässt sich Nachvollziehen als eine Tätigkeit verstehen, deren sich schon kleine Kinder bedienen, wenn sie auf ins Auge springende Merkmale des Verhaltens von etwas hinweisen, das sie gerade sehen oder gesehen haben, etwa indem sie angesichts einer durchs Gras gleitenden Schlange eine schlängelnde Handbewegung machen (vgl. dazu Plessner 1982). Ganz analog kann ich die Frage, was Pia gerade getan hat, dadurch beantworten, dass ich einfach das tue, was Pia getan hat, das heißt, indem ich etwas tue, das in relevanten Hinsichten dieselbe Form hat wie das, was Pia getan hat. Wenn mich nun jemand fragt, wie »Forgive « von Burial klingt, so kann ich versuchen zu beschreiben, was ich höre, und mich dabei durchaus auf Vergleiche mit anderer Musik oder Assoziationen stützen; und ich kann die Form dessen, was ich höre, artikulieren, indem ich mich beispielsweise körperlicher, das heißt tänzerischer oder gestischer Bewegungen bediene und darauf vertraue, dass sich dem Adressaten der nachvollziehenden Bewegung die Pointe der Bewegung vor dem Hintergrund seines propriozeptiven Erfahrungsschatzes mitteilt - zumindest so, dass er beim Hören des Stücks so etwas sagt wie: »Ah, ich glaub', ich verstehe, was du meinst.«

Mittels solcher Bewegungen sage ich natürlich nicht, wovon das Musikstück handelt oder was es repräsentiert, ich versuche vielmehr mitzuteilen, wie ich es erfahre. Ich artikuliere gewissermaßen - um in loser Verbindung

16 Hier liegt der Einwand nahe, dass die Musik ja zum Beispiel Emotionen ausdrücke und insofern doch repräsentationalen Gehalt habe. Wie schwierig es ist, diese Intuition zu verteidigen, macht schon die Frage deutlich, wie genau dieser repräsentationale Gehalt zu bestimmen wäre. Drückt die Musik die (gegenwärtigen, vergangenen?) Emotionen der Komponistin, des Sängers oder des Bassisten aus? Und wenn sie nicht die Emotionen dieser Leute ausdrückt, wessen Emotionen dann? Darüber hinaus haben Emotionen meist formale Objekte, etwas worauf sie sich beziehen, und man fragt sich, vermittels welcher Ressourcen Musik in der Lage sein könnte, beispielsweise einen Sachverhalt zu bestimmen, auf den sich Furcht oder Neid richtet. Einen ausgezeichneten Überblick über das Verhältnis zwischen Musik und Emotionen gibt Zwinggi (2016: Kap. 1).

17 Detaillierte Vorschläge zur Erläuterung des Verstehens von Musik in Begriffen des Nachvollzugs finden sich in Becker (2007) sowie in Vogel (2007). 
zu Gottlob Frege zu reden - die Gegebenheitsweise, in der mir das Stück qua meines Nachvollzugs gegenübersteht und damit seinen Sinn. Wenn ich den Sinn artikulieren will, den ich vermittels eines Nachvollzugs, also durch eine ausgeführte oder imaginierte Tätigkeit oder mittels einer Vorstellung erfasst habe, bin ich also nicht darauf festgelegt, sprachlich artikulierte Begriffe zu verwenden. Mein Verstehen scheitert insofern nicht daran, dass ich das Spezifische des Stücks nicht in Worte fassen kann oder sprachlich nicht artikulieren kann, warum meine Tätigkeit oder Vorstellung geeignet ist, den Sinn des Stücks zu erfassen und auszudrücken. Der Begriff des Nachvollzugs ist daher offen für eine Form des Verstehens, dessen Erfolgsbedingungen nicht erst dann erfüllt werden, wenn es gelingt, den Sinn sprachlich explizit zu machen. ${ }^{18}$

Am anschaulichsten lässt sich das Konzept des Nachvollzugs im Rekurs auf Tätigkeiten wie Gestikulieren oder Tanzen plausibel machen, aber es ist, wie ich bereits angedeutet habe, nicht darauf angewiesen, dass wir Nachvollzüge realisieren, indem wir tatsächlich körperliche Bewegungen vollziehen. Denn wir können unsere Wahrnehmung der Musik auch mit Hilfe imaginärer Bewegungen und Vorstellungen strukturieren, zu denen uns die Musik inspiriert. Wir können etwa einen Streicherklang als eine leuchtende Fläche hören oder eine Klangsequenz als Sturz (vgl. dazu Reuter 2015). Auch wenn uns manche solcher Vorstellungen aufgrund des kausalen Einflusses des Stücks auf uns näherliegen als andere, geht es hier nicht um bloß kontingente Assoziationen, sondern um Tätigkeiten und Vorstellungen, die geeignet sind, jene Klangereignisse, die mit dem Erklingen eines Musikstücks einhergehen, in einen Zusammenhang zu bringen, und zwar in einen, der verständlich macht, warum sich das Musikstück aus ebendiesen Klangereignissen zusammensetzt.

Musikstücke sind, abgesehen vielleicht von aleatorischen, keine bloßen Ansammlungen von Klangereignissen, und es liegt auf der Hand, dass jemand, der ein Musikstück versteht, den Zusammenhang der wahrgenommenen Klänge erfahrend erfasst haben muss. Er muss nicht das Konstruktionsprinzip angeben können, das der Komposition des Stücks zugrunde liegt, aber er muss es auf eine Weise hören können, vermöge deren die Klangereignisse zu einer strukturierten Erfahrung beitragen. Verstehenden Hörern muss sich der Zusammenhang der wahrgenommenen Klangereignisse in der Erfahrung erschließen. Angesichts dieser Aufgabe besteht die Idee des Nachvollzugs darin, dass eine Sequenz von Klangereignissen Sinn gewinnt, indem man sie nach dem Modell einer zusammenhängenden Bewegung erfährt, einer Bewegung, deren

18 Dass die Erfolgsbedingungen ästhetischen Verstehens relativ offen sind, stellt meines Erachtens ein zentrales Element für die Erklärung der ästhetischen Lust bereit. Vgl. dazu meine Ausführungen in Vogel (2013: Abschnitt III). 
Einheit uns ebenso unmittelbar verständlich ist, wie die Einheit einer ablehnenden Geste, eines Sonnenaufgangs, eines Sturzes oder einer Umarmung (vgl. dazu Becker 2007: 289f.). Da wir gewissermaßen Experten darin sind, den Anfang und das Ende von Handlungen oder, allgemeiner, die Einheit von körperlichen Bewegungen (insbesondere solchen, die Handlungen sind) zu erfassen, ${ }^{19}$ können wir Musik mit Hilfe solcher Bewegungen strukturieren und in der Erfahrung Einheit verleihen.

Darüber hinaus ist aber die Musik selbst durch Bewegungskategorien bestimmt: Klänge und Töne sind für uns in der Vertikale des musikalischen Raums auf unterschiedlichen Höhen positioniert, sodass wir Klangfolgen als auf- oder absteigend hören oder als verharrend. ${ }^{20}$ Zugleich sind die Klangereignisse horizontal nicht in einer Dimension bloßer Sukzession situiert, sondern in der musikalischen Zeit, die durch Ordnungen wie Metrum und Takt sowie durch rhythmische Gewichtungen strukturiert ist (vgl. dazu genauer Vogel 2018: Abschn. 3). Vorstellungen körperlicher Bewegungen liegen uns beim Musikverstehen also auch deshalb nahe, weil wir Musik selbst als etwas hören, das durch Kategorien der Bewegung bestimmt ist.

\section{Musik, Nachvollzug, Haltung, Person}

Die Weise, in der Menschen ihre Körper bewegen, welcher Habitus ihnen zu eigen ist, ist wesentlich durch das bestimmt, was sie tun und wie sie tun, was sie tun. Jemandem, der als Model arbeitet oder professionell in einer Ballettkompanie tanzt, sind die tausendfach eingeübten Körperbewegungen genauso zur zweiten Natur geworden, wie jemandem, der seine Brötchen als Fahrradbote im Verkehr einer Großstadt verdient. Während sich die Bewegungen von

19 Die Einheit von Handlungen können wir im Rekurs auf explizite, kausal relevante Intentionen erläutern oder, wie Michael Thompson vorgeschlagen hat, als die von Prozessen, deren Phasen ihrer Einheit dienen, so dass zwischen ihnen ein teleologischer Nexus besteht, der sich in einer Kette von Warum-Fragen entfalten lässt: Warum schält Karl Kartoffeln? Weil er Pellkartoffeln kocht. Warum kocht er Pellkartoffeln? Weil er einen Kartoffelsalat macht. Warum macht er Kartoffelsalat? Weil er einen Kindergeburtstag vorbereitet. Und so fort, vgl. Thompson (2011).

20 Man kann darüber streiten, ob die Rede von »hoch« und »tief« zur Charakterisierung von Tonhöhen genuin metaphorisch ist. Es scheint mir jedoch klar zu sein, dass Hörer unabhängig davon, welche Ausdrücke ihre jeweilige Sprache dafür bereitstellt (das Englische »high « und »low«, das Französische »grave « und »aigu«) musikalische Bewegungen als auf- oder abwärtsgerichtet hören. Das jedoch kann nur verständlich gemacht werden, wenn die Klänge am Anfang der Bewegung relativ zu den Endklängen als höher oder tiefer gehört werden; vgl. dazu Scruton (1997: 21). 
Models oder Balletttänzern (wie die von Angehörigen der höfischen Gesellschaft oder des »gehobenen« Bürgertums, vgl. Bourdieu 1982) auch an expliziten Bewegungsidealen orientieren, stellen sich die Bewegungsmuster und Haltungen von Fahrradboten eher infolge einer Reaktion auf die Arbeitsbedingungen ein, in denen die permanente Übersicht über die Bewegungen anderer Verkehrsteilnehmer sowie ein hochgetaktetes Bewusstsein für knappe Zeitressourcen eine Körperspannung erzeugen, die nicht nur den Tritt, sondern auch die Körperhaltung des Fahrradkuriers bestimmen.

Wenn es richtig ist, dass viele unserer Nachvollzüge beim Musikhören tatsächlich Handlungen sind (wie im Falle des Tanzens) oder Tätigkeiten imaginieren, und wenn Handlungen oder Tätigkeiten körperliche und psychische Voraussetzungen haben - etwa in Form spezifischer Spannungszustände oder Aufmerksamkeitsmuster -, dann scheint mir das Beispiel des Fahrradboten gut geeignet zu sein, um verständlich zu machen, wie Verstehensherausforderungen, die von (Pop-)Musik ausgehen, ${ }^{21}$ auf die Strukturierung unserer Körperbewegung (Hexis) und auf unsere Einstellung der Welt gegenüber (Habitus) Einfluss nehmen können.

Wenn uns Musik vor die Aufgabe stellt, ihre Merkmale als Momente einer Einheit zu erfassen, und Körperbewegungen nicht zuletzt aufgrund der Bewegungskategorien, die für Musik grundlegend sind, privilegierte Nachvollzugsmodelle darstellen, dann ist klar, dass Musik vermöge der Nachvollzugsmodelle, die sie uns nahelegt, Einfluss darauf nimmt, wie wir unsere Aufmerksamkeit organisieren, welche Körperspannungen wir bereitstellen, um die Nachvollzüge zu realisieren, und wie wir uns halten.

In diesem Sinne stellt ein Drum and Bass-Track mit 162 bpm wie Goldies »Kemistry« eine Herausforderung für unsere Nachvollzugskompetenzen dar, die der Herausforderung verwandter ist, welche vom Zeitdruck und der Komplexität des innerstädtischen Verkehrs für die Navigationskompetenzen des Radkuriers ausgeht, als die Herausforderung, die von einem Ambientstück wie dem oben erwähnten »Kobresia« von Biosphere ausgeht.

21 Herausforderungen vermag Roger Scruton im Fall der Pop-Musik nicht zu sehen, unter anderem, weil er nicht verstanden hat, dass beispielsweise Sound genauso ein essentieller Parameter von Pop-Musik ist, wie agogische Nuancierungen, die partiell gerade auf den regelmäßigen Beat angewiesen sind, den Scruton nur als Verfallssyndrom deuten kann. Der Vorwurf der Formlosigkeit, der immer wieder in Stellung gebracht wird, um sein negatives Diktum über Pop-Musik zu begründen, kann genauso gut als Ausdruck der Unfähigkeit verstanden werden, relevante Aspekte von Pop-Musik als Aspekte einer spezifischen musikalischen Einheit zu hören; vgl. Scruton (1997: 496-502). 
Wenn man eine Antwort auf die Herausforderung des Musikstücks findet und der Nachvollzug darüber hinaus lustvoll ist, dann organisiert man die eigene Wahrnehmungs- und Strukturierungsfähigkeit auf eine Weise, die nicht ohne Einfluss darauf bleiben kann, wie man den innerstädtischen Verkehr oder allgemeiner die Bedingungen wahrnimmt, unter denen man lebt. Das aber läuft darauf hinaus, der Lebenswelt als eine anders beschaffene Person gegenüberzustehen. Genki78 hatte die Faszination, die von »Melt « ausging, wenig erhellend auf den großartigen Sound zurückgeführt, ohne zu erläutern, was daran faszinierend war. Fizmaster hingegen verleiht seiner/ihrer Begeisterung für Leftism großformatig und gewissermaßen im Geiste Hegels Ausdruck: »This album is a true celebration of what it is to be alive and human in the late 20th century $\ll 22$

Wenn die vorangegangene Analyse des Verhältnisses zwischen Nachvollzügen und Haltungen nicht fehlgeht, ${ }^{23}$ dürfen wir Fizmasters Lob in folgendem Sinne deuten: Leftism ist ein Album, dessen Rezeption als Einheit einer Aufmerksamkeitsorganisation bedarf, die der verwandt ist, vermittels derer sich menschliche Wesen im ausgehenden 20sten Jahrhundert orientieren (können). Darüber hinaus regt seine fast durchgängig tanzbare rhythmische Strukturierung Nachvollzüge an, die lustvoll erfahren werden können. Wenn Fizmasters Diktum etwas Zutreffendes sagt, müssen wir zudem unterstellen, dass das ausgehende Jahrhundert aus der Perspektive der Erfahrung durch Merkmale geprägt ist, die denen der Musik ähneln. Leftism ist nun nicht einfach ein Progressive House-Album, sondern umfasst eine große Bandbreite von Einflüssen, insbesondere aus Tribal, Dub, Reggae, HipHop, Trance und Jungle. Aufgrund dieser unterschiedlichen Einflüsse stellt sich die Frage nach einem inneren Zusammenhang des Albums, ${ }^{24}$ wie sich die Frage nach dem Zusammenhang einer Welt stellt, in der Stammesgesellschaften und solche auf dem Weg zur postindustriellen Gesellschaft koexistieren, und es scheint, als würde der Zusammenhang des Albums über die musikalisch heterogenen

22 www.discogs.com/Leftfield-Leftism/master/66073, Zugriff: 10.4.2017.

23 Die Idee, Haltungen im Rekurs auf Nachvollzüge zu erläutern, geht auf Vogel (2007) zurück. Im Gegensatz zur Pose, die in Diederichsens Überlegungen eine zentrale Rolle spielt, ist eine Haltung weder etwas, das intentional markiert oder demonstrativ in den Fokus der Aufmerksamkeit gerückt wird, noch etwas, das gewohnheitsmäßig im Verdacht steht, nur vorgeblich zu sein.

24 Dass sich die Musiker mit diesem Problem auseinandergesetzt haben, machen die Zitate im Abschnitt »Produktion « auf https://en.wikipedia.org/wiki/Leftism_ (album) deutlich (Zugriff: 10.4.2017). 
Aspekte und Stile mittels des Tanzes gestiftet, also über eine nachvollziehende Bewegungsform, die Lebendigkeit feiert. ${ }^{25}$

Den Ausgangspunkt meiner Überlegung bildete der Verdacht, dass weder das quasi-pharmakologische Wissen, das Hörer hinsichtlich ihrer Selbstregulation im Umgang mit Musik erwerben, noch kontingente, mit Musik verknüpfte Erinnerungen verständlich machen können, warum Musik ein Medium ist, das eine wichtige Rolle im Kontext von Prozessen des Selbstverstehens spielen kann. Diese Rolle können wir vielmehr nur dann verstehen, wenn wir PopMusik als einen Gegenstand des Verstehens betrachten. Weil dieses Verstehen nicht als das Erfassen einer angebbaren Bedeutung erläutert werden kann, sondern als Nachvollzug, und Nachvollziehen, ein kreativer Prozess ist, in dem wir unser Erfahren und damit einen wesentlichen Aspekt unserer Person experimentell (re-)strukturieren, tragen Nachvollzüge nicht nur etwas zur Strukturierung ihrer Gegenstände, sondern auch zur Strukturierung ihrer Subjekte bei. Ein Mensch, dem es gelungen ist, anlässlich von Musik geeignete Nachvollzugsmodelle zu entwickeln - Modelle, die es ihm erlauben, die Einheit oder den Zusammenhang dieser Musik zu erfahren -, ist eine andere Person als der Mensch, der diese Nachvollzugsmodelle noch nicht entwickelt hat oder noch nicht entwickeln konnte. Er ist eine Person, der andere Mittel der Strukturierung der Wirklichkeit zur Verfügung stehen und die andere Haltungen der Wirklichkeit gegenüber einzunehmen vermag. Da Musik zudem ein Medium ist, das wesentlich durch Kategorien der Bewegung bestimmt ist, sodass strukturierende Nachvollzüge von Musik (reale oder imaginierte) Bewegungen und Haltungen des eigenen Körpers hervorbringen, scheint sie für Prozesse des Selbstverstehens besonders geeignet zu sein, insofern wir in diesen Nachvollzügen probehalber Körperbilder, Haltungen und Einstellungen

25 Mir ist wichtig zu betonen, dass der Zusammenhang zwischen gesellschaftlichen und musikalischen Strukturen, der hier ins Auge gefasst wird, deutlich vermittelter ist, als etwa in Ernst Blochs kruder Parallelisierung zwischen sozialen Schichten und musikalischen Stimmen: »Dem beginnenden Unternehmertum entsprechen die Herrschaft der melodieführenden Oberstimme und die Beweglichkeit der übrigen ebenso, wie der cantus firmus in der Mitte und die gestufte Vielstimmigkeit der ständischen Gesellschaft entsprochen haben « (Bloch 1977: 1249). Die Ähnlichkeit, die meine obige Analyse in Anspruch nimmt, ist eine zwischen Erfahrungen, die Personen mit musikalischen und sozialen Strukturen machen, und keine zwischen (intrinsischen) Eigenschaften musikalischer und sozialer Entitäten. Dabei ist die Möglichkeit einer wechselseitigen Strukturierung des Erfahrens, anders als in Blochs Vulgärmaterialismus nicht ausgeschlossen; vgl. dazu auch Kim (2013). 
einnehmen, die wir darauf prüfen, ob sie (auch im Kontext der sozialen Situierung) zu uns passen. ${ }^{26}$

Vor dem Hintergrund dieser Überlegungen wird im Versuch, Pop-Musik nachvollziehend zu verstehen, nicht nur thematisch, was das Spezifische der jeweiligen Musik ausmacht, sondern was uns ausmacht. Pop-Musik stellt uns Fragen des Typs: Will ich einer sein, der sich so und so bewegt? Der sich so hält oder in Szene setzt? Der solche Posen oder Haltungen oder ein bestimmtes, durch diese Haltung vermitteltes Verhältnis zur Welt einnimmt? Kurzum: Will ich eine Person sein, die sich zurechnen will, was ihr das Verstehen dieser Musik erlaubt? Der Pop-Musik-Komplex reichert diese Hinsichten durch eine Vielzahl von Aspekten wie Songtexte, Videos, Mode oder Stars an (Diederichsen 2014: XI), davon aber scheint mir die Fähigkeit der Pop-Musik, solche Fragen aufzuwerfen, nicht abzuhängen. ${ }^{27}$

\section{Literatur}

Adorno, Theodor W. (1978 [1956]). »Fragment über Musik und Sprache.« In: Gesammelte Schriften Bd. 16: Musikalische Schriften I-III. Hg. v. R. Tiedemann. Frankfurt/M.: Suhrkamp, S. 251-256.

Becker, Alexander (2007). »Wie erfahren wir Musik?« In: Musikalischer Sinn. Beiträge zu einer Philosophie der Musik. Hg. v. Alexander Becker u. Matthias Vogel. Frankfurt/M.: Suhrkamp, S. 265-313.

Bloch, Ernst (1977 [1959]). Das Prinzip Hoffnung Bd. 3, Frankfurt/M.: Suhrkamp.

Bourdieu, Pierre (1982 [1979]). Die feinen Unterschiede. Kritik der gesellschaftlichen Urteilskraft. Frankfurt/M.: Suhrkamp.

DeNora, Tia (1999). »Music as a Technology of the Self.«In: Poetics 27, S. 31-56.

Diederichsen, Diedrich (2014). Über Popmusik, Köln: Kiepenheuer \& Witsch.

Bob Dylan (2017). »Nobel Lecture«, http://www.nobelprize.org/nobel_prizes/liter ature/laureates/2016/dylan-lecture.html, Zugriff: 10.4.2017.

Hanslick, Eduard (1989 [1854]). Vom Musikalisch-Schönen. Ein Beitrag zur Revision der Ästhetik der Tonkunst, Wiesbaden: Breitkopf \& Härtel.

Huron, David (2001). »Is Music an Evolutionary Adaptation?«In: Annals of the New York Academy of Sciences 930, S. 43-61.

26 Auch der partiell scheiternde Versuch, Musik nachvollziehen, die sich wie beispielsweise der K-Pop südkoreanischer idol groups die Automatismen des Perzeptions-Aktions-Systems (PAS) zunutze macht, das zu unserer Fähigkeit zum vokalen Nachahmen beiträgt, kann noch als Entwicklung einer Haltung verstanden werden - einer Haltung, die sich in der Bewunderung von Stars niederschlägt, deren »prothetisch-superioristische « Stimmlichkeit mit einer transhumanen Perfektion spielt, die jenseits der Nachvollziehbarkeit vermittels eines bloß menschlichen Körpers liegt; vgl. dazu Meier (2014) sowie als Beispiel solcher Musik »Can't Nobody M/V« von 2NE1, www.youtube.com/watch? v=lhi_kJJj_8A, Zugriff: 10.4.2017

27 Für kritische Hinweise danke ich Norman Hammel, Anna Mense, Laura Schneider und Ralf von Appen. 
Kim, Jin Hyun (2013). "Shaping and Co-Shaping Forms of Vitality in Music: Beyond Cognitivist and Emotivist Approaches to Musical Expressiveness.«In: Empirical Musicology Review 8 (3-4), S. 162-173.

Meier, Niklas (2014). Warum werde ich ständig kurzatmig? Stimmakustische Semiotik in Produktionen südkoreanischer Idol Groups. « Unveröff. MA-Thesis, Institut für Musikwissenschaft Universität Leipzig.

Plessner, Helmuth (1982 [1951]). »Zur Anthropologie der Musik.«In: Gesammelte Schriften Bd. 7. Hg. v. Günther Dux, Odo Marquard u. Elisabeth Ströker, Frankfurt/M.: Suhrkamp 1982, S. 184-200.

Reuter, Gerson (2015). »Die Rolle der Vorstellungskraft für unsere Musikwahrnehmung: das Phänomen des Hören-als. « In: Deutsche Zeitschrift für Philosophie 63(4), S. 625-643.

Scruton, Roger (1997). The Aesthetics of Music. Oxford: Oxford University Press.

Thompson, Michael (2008). Leben und Handeln. Grundstrukturen der Praxis und des praktischen Denkens. Berlin: Suhrkamp.

Trevarthen, Colwyn (2000). »Musicality and the Intrinsic Motive Pulse: Evidence from Human Psychobiology and Infant Communication. «In: Musicae Scientiae 3, S. 155215.

Vogel, Matthias (2007). »Nachvollzug und die Erfahrung musikalischen Sinns. «In: Musikalischer Sinn. Beiträge zu einer Philosophie der Musik. Hg. v. Alexander Becker u. Matthias Vogel. Frankfurt/M.: Suhrkamp, S. 314-368.

Vogel, Matthias (2013). »Ästhetisches Erfahren - ein Phantom?« In: Kunst und Erfahrung. Beiträge zu einer philosophischen Kontroverse. Hg. v. Stefan Deines, Jasper Liptow u. Martin Seel, Berlin: Suhrkamp, S. 91-119.

Vogel, Matthias (2018). »Musik und Geste - Wahlverwandtschaft oder zufällige Liaison?« In: Geste und Musik. Theorien, Ansätze, Perspektiven. Hg. v. Katrin Eggers und Christian Grüny. Paderborn: Wilhelm Fink, S. 51-70.

Zwinggi, Stefan (2016). Musik als affektive Selbstverständigung. Eine integrative Untersuchung über musikalische Expressivität. Freiburg u. München: Karl Alber.

\begin{abstract}
It is perfectly legitimate to ask why we listen to music. While the answer »for its own sake « is at best a riddle, the reference to mere (pleasurable) effects is not specific enough. My claim is that an informative answer to this question has to take into account the fact that music is something we can understand. To make this claim plausible, I initially look at narratives in which people report that listening to pieces of (instrumental) pop music has changed their lives. Since material living conditions may not have been affected by these changes, we can assume that they have taken place in dimensions that play a role for the listeners' self-image. Against the background of a theory of understanding music in terms of re-enactment, I then try to make intelligible why pop music in particular is something we can value as a powerful medium for the development and transformation of self-images.
\end{abstract}

